(6) OPEN ACCESS

\title{
The contribution of viruses and bacteria to community-acquired pneumonia in vaccinated children: a case-control study
}

\author{
Mejbah Uddin Bhuiyan, ${ }^{1,2}$ Thomas L Snelling, ${ }_{1}^{2,3}$ Rachel West, ${ }_{1}{ }^{2}$ Jurissa Lang, ${ }_{1}{ }^{4}$ \\ Tasmina Rahman, ${ }^{2,5}$ Caitlyn Granland, ${ }^{2}$ Camilla de Gier, ${ }^{2,5}$ Meredith L Borland, ${ }^{1,6,7}$ \\ Ruth B Thornton, ${ }^{2,5}$ Lea-Ann S Kirkham, ${ }^{2,5}$ Chisha Sikazwe, ${ }^{4,5}$ Andrew C Martin, ${ }^{8}$ \\ Peter C Richmond, ${ }^{1,2,8}$ David W Smith, ${ }^{4,5}$ Adam Jaffe, ${ }^{9}$ Christopher C Blyth ${ }^{1,2,3}$
}

For numbered affiliations see end of article.

\section{Correspondence to}

Mejbah Uddin Bhuiyan, Division of Paediatrics, School of Medicine, Faculty of Health and Medical Sciences, The University of Western Australia, Perth WA 6009, Australia;

mejbah.bhuiyan@uwa.edu.au

Received 21 May 2018 Revised 12 September 2018 Accepted 17 September 2018

Published Online First

18 October 2018

\section{SLinked}

- http://dx.doi.org/10.1136/ thoraxjnl-2018-212625

Check for updates

(C) Author(s) (or their employer(s)) 2019. Re-use permitted under CC BY-NC. No commercial re-use. See rights and permissions. Published by BMJ.

To cite: Bhuiyan MU Snelling TL, West $R$, et al.

Thorax 2019:74:261-269.

\section{ABSTRACT}

Introduction Respiratory pathogens associated with childhood pneumonia are often detected in the upper respiratory tract of healthy children, making their contribution to pneumonia difficult to determine. We aimed to determine the contribution of common pathogens to pneumonia adjusting for rates of asymptomatic detection to inform future diagnosis, treatment and preventive strategies.

Methods A case-control study was conducted among children $<18$ years in Perth, Western Australia. Cases were children hospitalised with radiologically confirmed pneumonia; controls were healthy children identified from outpatient and local immunisation clinics. Nasopharyngeal swabs were collected and tested for 14 respiratory viruses and 6 bacterial species by Polymerase chain reaction (PCR). For each pathogen, adjusted odds ratio $(\mathrm{aOR} ; 95 \% \mathrm{Cl}$ ) was calculated using multivariate logistic regression and population-attributable fraction $(95 \% \mathrm{Cl})$ for pneumonia was estimated.

Results From May 2015 to October 2017, 230 cases and 230 controls were enrolled. At least one respiratory virus was identified in $57 \%$ of cases and $29 \%$ of controls (aOR: $4.7 ; 95 \% \mathrm{Cl}: 2.8$ to 7.8 ). At least one bacterial species was detected in $72 \%$ of cases and $80 \%$ of controls (aOR: $0.7 ; 95 \% \mathrm{Cl}: 0.4$ to 1.2 ). Respiratory syncytial virus (RSV) detection was most strongly associated with pneumonia (aOR: 58.4; 95\% Cl: 15.6 to 217.5). Mycoplasma pneumoniae was the only bacteria associated with pneumonia (aOR: $14.5 ; 95 \% \mathrm{Cl}: 2.2$ to 94.8). We estimated that RSV, human metapneumovirus (HMPV), influenza, adenovirus and Mycoplasma pneumoniae were responsible for $20.2 \%$ (95\% Cl: 14.6 to 25.5$), 9.8 \%(5.6 \%$ to $13.7 \%), 6.2 \%$ (2.5\% to $9.7 \%)$, $4 \%(1.1 \%$ to $7.1 \%)$ and $7.2 \%$ (3.5\% to $10.8 \%)$ of hospitalisations for childhood pneumonia, respectively. Conclusions Respiratory viruses, particularly RSV and $\mathrm{HMPV}$, are major contributors to pneumonia in Australian children

\section{BACKGROUND}

Pneumonia remains as a leading cause of hospitalisation and death among children worldwide, with nearly 120 million new cases and one million deaths annually, mostly among children aged under 5 years. ${ }^{1}$ Although the greatest disease burden is

\section{Key messages}

What is the key question?

- How much do respiratory viruses and bacteria contribute to community-acquired pneumonia in highly vaccinated children?

What is the bottom line?

- Many pneumonia-causing pathogens are also detected in asymptomatic children, thereby confounding our understanding to their contribution to pneumonia in children.

Why read on?

- We conducted the largest age-frequency matched case-control study in a developed setting showing substantial contribution of respiratory syncytial virus, human metapneumovirus and Mycoplasma pneumoniae to all childhood pneumonia requiring hospitalisation in the post pneumococcal conjugate vaccine era.

in developing countries, pneumonia remains an important public health concern in developed countries where concerns are compounded by growing antibiotic resistance, replacement disease caused by vaccine-escape strains and the emergence of newly identified pathogens. ${ }^{23}$ In Australia, there are two to eight pneumonia hospitalisations per 1000 childyears among children $<5$ years old; Aboriginal children have more than a 10 -fold higher risk than non-Aboriginal children. ${ }^{45}$

Globally, bacteria, particularly Streptococcus pneumoniae and encapsulated and non-encapsulated strains of Haemophilus influenzae, are considered as the primary cause of pneumonia in young children. ${ }^{3}$ In recent years, there has been an increased focus on the role of respiratory viruses in childhood pneumonia, partly due to the reduction in bacterial disease associated with conjugate pneumococcal and Hib vaccine use ${ }^{67}$ and the increased capacity to detect virus through molecular diagnostic methods. These sensitive techniques have enabled detection of viruses in up to $92 \%$ of respiratory specimens of children with pneumonia. ${ }^{8-12}$ Many of the commonly detected respiratory viruses 
and bacteria are also detected in the nasopharynx of apparently healthy children, obscuring their exact contribution to childhood pneumonia. ${ }^{9} 11$ 13-15 Except for influenza vaccine, no licensed vaccines for common respiratory viruses are currently available although a number of vaccines for other respiratory viruses are in clinical trials. ${ }^{16-18}$

To date, few pneumonia aetiology studies from high-income settings seeking to quantify the exact contribution of pathogens to pneumonia have included healthy controls. ${ }^{911}$ Well-controlled epidemiological studies have reported the contribution of respiratory viruses to childhood pneumonia in developing settings, but their findings are unlikely to be generalisable to settings with better developed health infrastructure, including better vaccine coverage. ${ }^{151920}$ We conducted a prospective case-control study to estimate the contribution of respiratory viruses and bacteria to childhood pneumonia in West Australian (WA) children, a highly vaccinated and high-income population.

\section{METHODS}

This prospective case-control study was conducted from May 2015 through October 2017 following a published study protocol. $^{21}$

\section{Study population and data collection}

Cases were children aged $<18$ years old who presented with community-acquired pneumonia (CAP) to the Princess Margaret Hospital (PMH), the only tertiary paediatric hospital for the state of Western Australia (population: 2.6 million $^{22}$ ). WA children are highly vaccinated: pneumococcal conjugate vaccine (PCV) is funded on the national immunisation programme and coverage among infants is $>90 \%$. Seasonal influenza vaccine is also funded for WA children aged 6 months to $<5$ years and for older children with high-risk conditions. ${ }^{23}$

A diagnosis of CAP required: (1) the presence of acute respiratory symptoms (with or without fever) and (2) evidence of infiltrates or consolidation on chest X-ray. ${ }^{24} \mathrm{~A}$ pragmatic definition of radiologically confirmed pneumonia (infiltrates or alveolar consolidation as determined by the treating clinician) facilitating recruitment after hours (ie, when a radiologist is not available to review the chest X-ray). An equal number of contemporaneous healthy controls attending hospital outpatient orthopaedics or allergy clinics or a large urban community immunisation clinic were enrolled. Children attending clinic with respiratory symptoms, following recent respiratory illnesses or those previously enrolled as a case or control were not eligible. Controls were enrolled concurrently with cases throughout the study period, with the target enrolment in each of four age groups ( $\leq 1$ year, $1-5$ years, 6-9 years and $\geq 10$ years) frequency-matched to the number of cases enrolled in the preceding 2 weeks. The sample size calculation for the study has been previously described. ${ }^{21}$ A structured questionnaire was administered to parents/guardians to capture demography, relevant comorbidities including immunodeficiency, immunocompromised condition, congenital chromosomal abnormality, chronic respiratory, neuromuscular, neurological and cardiac disease and solid organ transplantation.

\section{Specimen collection and microbiological assays}

A flocked nasopharyngeal swab (FLOQSwabs; Copan Diagnostics, Murrieta, California, USA) was collected from each participant by a trained research assistant; for cases, the swab was collected $<36$ hours after hospital presentation. After collection, the swab was placed immediately into $1 \mathrm{~mL}$ Skim-Milk-Tryptone-Glucose-Glycerol-Broth on ice. All specimens were stored at $-80^{\circ} \mathrm{C}$ prior to nucleic acid extraction. A total of 14 viruses (influenza $\mathrm{A} / \mathrm{H} 1 \mathrm{~N} 1$ and $\mathrm{A} / \mathrm{H} 3 \mathrm{~N} 2$, influenza $\mathrm{B}$, respiratory syncytial virus (RSV), human parainfluenza virus (HPIV) type 1-3, human metapneumovirus (HMPV), adenovirus, rhinovirus (RV), human coronavirus (HCoV) types HCoVOC43, HCoV229E, HCoVHKU1 and HCoVNL63 and 6 bacteria (S. pneumoniae, Staphylococcus aureus, Moraxella catarrhalis, H. influenzae, Mycoplasma pneumoniae and Chlamydophila pneumoniae) were detected using polymerase chain reaction (PCR). Detailed procedures for laboratory testing for nucleic acid extraction and the primers and probes used in PCR have been described previously. ${ }^{21} \mathrm{RV}$ genotyping was done following published molecular methods. ${ }^{25}$

\section{Data management and analysis}

The crude odds ratio (OR) of exposure was calculated to assess the relative frequency of detection of each respiratory pathogen among cases and controls. Multivariate logistic regression was used to calculate the adjusted OR (aOR) of detection of each pathogen for cases versus controls, adjusting for underlying demographic differences, recent exposure to antibiotics and for the presence of other pathogens. The aOR for each pathogen was then used to estimate the population-attributable fraction (PAF). ${ }^{26}$ Analyses were performed using STATA software, V.13.0, and figures were produced using GraphPad Prism, Version 5.0 (GraphPad Software, California, USA).

\section{Ethical consideration}

Written informed consent was obtained from parents/legal guardians of all study participants. Assent was obtained from participants $\geq 7$ years old.

\section{RESULTS}

\section{Characteristics of study population}

We enrolled 230 children with pneumonia and 230 controls. Of 230 cases, $24(10 \%)$ cases were diagnosed with a parapneumonic effusion with 21 (9\%) parapneumonia effusions drained. All drained effusions had microscopic purulence consistent with empyema. Cases were hospitalised for a median of 2 days (IQR: 1-3); no deaths were observed. Compared with controls, a higher proportion of cases were Aboriginal, born premature and reported a smoker in the household ( $p<0.001$ for each) (table 1$)$. The proportion of respondents $<5$ years attending day care was $59.1 \%$ among cases $(n=87)$ and $36.6 \%$ among controls $(n=66)$, and the median days of attendance per week for cases was 3 days (IQR: 2-4) and for controls was 2 days (IQR: 2-3). A higher proportion of cases had comorbidities compared with controls $(14.7 \%$ vs $4.3 \%, \mathrm{p}<0.001)$. More cases than controls had antibiotics in the 7 days prior enrolment $(47.3 \%$ vs $2.1 \%, \mathrm{p}<0.001)$. The proportion of cases and controls who had received at least two doses of PCV was $91.3 \%$ and $87.8 \%$, respectively, and $10.4 \%$ of both cases and controls had received influenza vaccine in the preceding influenza season.

\section{Respiratory viruses and bacteria associated with pneumonia} Of 230 cases and 230 controls, one or more respiratory viruses were identified in $56.5 \%$ and $28.6 \%$, respectively (OR: 3.2; 95\% CI: 2.1 to 4.7 ), and at least one bacterial species was detected in $71.7 \%$ and $79.5 \%$, respectively (OR: 0.6 ; 95\% CI: 0.4 to 1.0$)$. The distribution of respiratory viruses and bacteria among cases and controls is summarised in table 2. Detection of both viruses and bacteria was observed in 104 cases (45.2\%) and in 56 controls $(24.3 \%)$. Cases were more likely than controls to 
Table 1 Characteristics of children with community-acquired pneumonia (cases) and healthy children (controls), Perth, Western Australia, May 2015-October 2017

\begin{tabular}{|c|c|c|c|}
\hline Parameter & $\begin{array}{l}\text { Case }(\%), \\
(n=230)\end{array}$ & $\begin{array}{l}\text { Control }(\%), \\
(n=230)\end{array}$ & $\begin{array}{l}\text { Total }(\%), \\
(\mathrm{N}=460)\end{array}$ \\
\hline \multicolumn{4}{|l|}{ Demographic and clinical data } \\
\hline \multicolumn{4}{|l|}{ Age } \\
\hline$\leq 12$ months & $21(9.1)$ & $23(10)$ & $44(9.5)$ \\
\hline $1-5$ years & $126(54.7)$ & $157(68.2)$ & $283(61.5)$ \\
\hline $6-9$ years & $60(26.1)$ & $27(11.7)$ & $87(18.9)$ \\
\hline $10+$ years & $23(10)$ & $23(10)$ & $46(10)$ \\
\hline Male sex & $120(52.1)$ & $122(53.0)$ & $242(52.6)$ \\
\hline Aboriginal* & $21(9.1)$ & $2(0.8)$ & $23(5.0)$ \\
\hline Premature* & $32(13.9)$ & $17(7.3)$ & $49(10.6)$ \\
\hline Smoker at household* & $38(16.5)$ & $22(9.5)$ & $60(13.0)$ \\
\hline \multicolumn{4}{|l|}{ Existing health conditions $†$} \\
\hline Any comorbidity & $34(14.7)$ & $10(4.3)$ & $44(9.5)$ \\
\hline Immunodeficiency & $7(3.0)$ & $1(0.4)$ & $8(1.7)$ \\
\hline $\begin{array}{l}\text { Immunocompromised } \\
\text { condition }\end{array}$ & $5(2.1)$ & $0(0.0)$ & $5(1.1)$ \\
\hline $\begin{array}{l}\text { Congenital chromosomal } \\
\text { abnormality }\end{array}$ & $16(6.9)$ & $4(1.7)$ & $20(4.3)$ \\
\hline Chronic respiratory illness & $9(3.9)$ & $2(0.8)$ & $11(2.3)$ \\
\hline $\begin{array}{l}\text { Chronic neuromuscular } \\
\text { disorder illness }\end{array}$ & $9(3.9)$ & $2(0.8)$ & $11(2.3)$ \\
\hline
\end{tabular}

${ }^{*} P$ value $<0.001$ for difference between cases and controls.

tNo child had an organ transplant, one case had chronic cardiac disease and one case had an intracranial shunt.

have multiple (two or more) viruses detected (OR: 5.1, 95\% CI: 1.7 to 15.1 ) but not multiple bacterial species (OR: 1.0, 95\% CI: 0.7 to 1.4$)$.

\section{Viral pathogens}

The multivariate logistic regression showed that after adjusting for demographic differences, antibiotic exposure in the preceding 7 days, and presence of other pathogens, RSV, HMPV, influenza virus and adenovirus were detected significantly more often among cases than controls with aOR 58.4 (95\% CI: 15.6 to 217.5 ), 37.2 (95\% CI: 7.8 to 177.7$), 10.1$ (95\% CI: 1.8 to 57.1) and 12.1 (95\% CI: 1.4 to 104.4 ), respectively (table 2). None of the influenza-positive cases $(n=16)$ or controls $(n=3)$ had received influenza vaccine during the preceding influenza season. RV speciation was available for 57 (69\%) of 83 positive samples (26 cases; 31 controls). RV-A was detected in 13 cases (50\%) and 15 controls (48.3\%), RV-B in 1 (3.8\%) case and 2 $(6.4 \%)$ controls and RV-C was detected in $12(46.1 \%)$ cases and 14 (45.1\%) controls.

Respiratory viruses were detected more commonly among children $<5$ years compared with other age groups (figure 1). More than 75\% of cases positive for RSV, HPIV, HMPV and adenovirus were aged less than 3 years. Respiratory viruses were detected throughout the year with frequent detection during the Australian winter months (June to August). RV was detected throughout the year, while RSV, influenza and HMPV were mostly detected between May and November and HPIV between December and April; virus distribution among cases and control children was similar along the year calendar (figures 2 and 3).

\section{Bacterial pathogens}

The multivariate logistic regression showed M. pneumoniae were detected significantly more often among cases than controls with aOR 14.5 (95\% CI: 2.2 to 94.8). The frequency of detection of other bacteria was similar among cases and controls (table 2).

Nineteen $(32.1 \%)$ of 59 cases with $S$. pneumoniae detected had prior antibiotic exposure compared with 1 (1.6\%) of 61 controls; for $H$. influenzae, the proportion was $45.3 \%(34 / 75)$ in cases and 3.2\% (2/61) in controls; for M. catarrhalis, $29.1 \%$ $(30 / 103)$ in cases and $2.0 \%(3 / 144)$ in controls; for $S$. aureus, $36.8 \%(14 / 38)$ in cases and $0 \%(0 / 36)$ in controls and for $M$. pneumoniae, $89.4 \%(17 / 19)$ in cases and $0 \%(0 / 1)$ in controls. Given the potential for antibiotics to influence the detection of bacterial pathogens, the analysis was repeated including only those with no prior antibiotic exposure: apart from $M$. pneumoniae (aOR 16.7; 95\% CI: 1.6 to 164.8), no significant difference in S. pneumoniae (aOR 0.6; 95\% CI: 0.2 to 1.3 ), H. influenzae (aOR 0.9; 95\% CI: 0.4 to 1.8 ), M. catarrhalis (aOR 0.7; 95\% CI: 0.4 to 1.4 ) and $S$. aureus (aOR $1.5 ; 95 \% \mathrm{CI}: 0.7$ to 3.5 ) detection was noted.

Among children with no prior antibiotic exposure, few differences were noted in the age-group distribution of bacterial detection (figure 1). Among infants (aged $<1$ year), more cases than controls were positive for $S$. pneumoniae $(6 / 15,40 \%$ vs $2 / 23$, $8.7 \%, \mathrm{p}=0.02)$. M. pneumoniae was detected more commonly in school-aged children than in younger children. M. catarrhalis was detected throughout the year, whereas $S$. pneumoniae, $H$. influenzae, S. aureus and M. pneumoniae were detected more commonly in winter months (figures 4 and 5).

\section{Population-attributable fraction}

We estimated that detectable respiratory viruses are responsible for 44.4\% (95 \% CI: $33.8 \%$ to $53.3 \%$ ) of all childhood pneumonia in Western Australia (table 2). RSV contributed nearly half of this burden $(20.2 \%$, 95\% CI: $14.6 \%$ to $25.5 \%)$ with HMPV contributing $9.8 \%$ (95\% CI: $5.6 \%$ to $13.7 \%$ ), influenza $6.2 \%$ (95\% CI: $2.5 \%$ to $9.7 \%$ ) and adenovirus $4.0 \%$ (95\% CI: $1.1 \%$ to $7.1 \%)$. The only detectable bacteria that could be shown to make a significant contribution to childhood pneumonia in our population was M. pneumoniae (7.2\%, 95\% CI: 3.5\% to $10.8 \%$ ). We were not sufficiently powered to estimate age group-specific PAF for these pathogens.

\section{DISCUSSION}

Respiratory viruses appear to contribute significantly to childhood pneumonia in Western Australia. By prospectively enrolling and testing children hospitalised with radiologically confirmed pneumonia and contemporaneous healthy children, we were able to assess the contribution of common respiratory viruses and bacteria to pneumonia adjusting for the background prevalence of detection in children without pneumonia. We estimate that detectable respiratory viruses contribute approximately $44 \%$ of all childhood pneumonia requiring hospitalisation.

Respiratory viruses were detected in the nasopharynx of $57 \%$ of children with pneumonia, which is comparable to the frequency of virus detection in other paediatric pneumonia studies. ${ }^{1127-29}$ Similar to previous studies, we were also able to detect respiratory viruses in the nasopharynx of nearly one-third of controls. ${ }^{11} 13{ }^{30}$ In another matched case-control study, viruses were detected more frequently (in $86 \%$ of cases and $55 \%$ of controls); that study only enrolled children during winter months when respiratory virus circulation is likely to be greater. ${ }^{9}$ 
Table 2 Distribution of respiratory pathogens in children with CAP (cases) and healthy children (controls), unadjusted and adjusted OR and population-attributable fraction for each respiratory pathogen, Perth, Western Australia, May 2015-October 2017

\begin{tabular}{|c|c|c|c|c|c|}
\hline Parameter & $\begin{array}{l}\text { Case }(\%), \\
(n=230)\end{array}$ & $\begin{array}{l}\text { Control (\%), } \\
(n=230)\end{array}$ & $\begin{array}{l}\text { Unadjusted OR } \\
(95 \% \mathrm{Cl})\end{array}$ & $\begin{array}{l}\text { Adjusted OR } \\
(95 \% \mathrm{Cl})^{*}\end{array}$ & $\begin{array}{l}\text { Population-attributable } \\
\text { fraction estimate, } \% \\
(95 \% \mathrm{Cl})\end{array}$ \\
\hline \multicolumn{6}{|l|}{ Respiratory virus } \\
\hline Influenza (any) & $16(6.9)$ & $3(1.3)$ & 5.6 (1.6 to 19.6$)$ & $10.1(1.8$ to 57.1$)$ & 6.2 (2.5 to 9.7$)$ \\
\hline Influenza A H1N1 & $4(1.7)$ & $1(0.4)$ & 4.0 (0.4 to 36.5$)$ & 23.1 (1.2 to 442.6$)$ & \\
\hline Influenza A H3 & $9(3.9)$ & $1(0.4)$ & 9.3 (1.1 to 74.2$)$ & $9.6(0.7$ to 119.7$)$ & \\
\hline Influenza B & $3(1.3)$ & $1(0.4)$ & 3.0 (0.3 to 29.3 ) & 2.7 (0.1 to 43.9$)$ & \\
\hline RSV & $46(20.0)$ & $3(1.3)$ & 18.9 (5.7 to 61.8$)$ & 58.4 (15.6 to 217.5$)$ & 20.2 (14.6 to 25.5$)$ \\
\hline HPIV (any) & $11(4.7)$ & 0 & - & - & - \\
\hline HPIV1 & $6(2.6)$ & 0 & - & - & - \\
\hline HPIV2 & 0 & 0 & - & - & - \\
\hline HPIV3 & $5(2.1)$ & 0 & - & - & - \\
\hline Rhinovirus & $35(15.2)$ & $48(20.8)$ & 0.7 (0.4 to 1.1$)$ & 1.3 (0.6 to 2.6$)$ & $3.6(-0.05$ to 12.2$)$ \\
\hline HMPV & $23(10)$ & $2(0.8)$ & $12.6(2.9$ to 54.3$)$ & $37.2(7.8$ to 177.7$)$ & 9.8 (5.6 to 13.7$)$ \\
\hline Adenovirus & $10(4.3)$ & $3(1.3)$ & 3.4 (0.9 to 12.6$)$ & 12.1 (1.4 to 104.4$)$ & $4(1.1$ to 7.1$)$ \\
\hline Coronavirus (any) & $8(3.4)$ & $11(4.7)$ & 0.7 (0.2 to 1.8$)$ & 0.8 (0.1 to 4.4$)$ & \\
\hline Coronavirus $\mathrm{OC} 43$ & $5(2.1)$ & $3(1.3)$ & 0.6 (0.1 to 2.5$)$ & $0.7(0.1$ to 3.9$)$ & \\
\hline Coronavirus NL63 & $4(1.7)$ & $3(1.3)$ & $1.3(0.3$ to 6.0$)$ & $1.5(0.1$ to 21.8$)$ & \\
\hline Coronavirus HKU1 & $1(0.4)$ & $2(0.8)$ & 0.5 (0.04 to 5.5$)$ & 0.5 (0.0 to 2724.1$)$ & \\
\hline Coronavirus 229E & 0 & $1(0.4)$ & - & - & \\
\hline At least one respiratory virus identified & $130(56.5)$ & $66(28.6)$ & $3.2(2.1$ to 4.7$)$ & 4.7 (2.8 to 7.8$)$ & 44.4 (33.8 to 53.3$)$ \\
\hline One virus & $111(48.2)$ & $62(26.9)$ & & & \\
\hline Two or more viruses & $19(8.2)$ & $4(1.7)$ & & & \\
\hline \multicolumn{6}{|l|}{ Bacteria } \\
\hline Streptococcus pneumoniae & $59(25.3)$ & $61(26.5)$ & $0.9(0.6$ to 1.4$)$ & $0.6(0.3$ to 1.4$)$ & \\
\hline Haemophilus influenzae & $75(32.6)$ & $61(26.5)$ & $1.3(0.8$ to 2.0$)$ & $0.9(0.4$ to 1.7$)$ & \\
\hline Moraxella catarrhalis & $103(44.7)$ & $144(62.6)$ & $0.4(0.3$ to 0.7$)$ & $0.6(0.3$ to 1.1$)$ & \\
\hline Staphylococcus aureus & $38(16.5)$ & $36(15.6)$ & $1.0(0.6$ to 1.7$)$ & 1.3 (0.6 to 2.9$)$ & \\
\hline Mycoplasma pneumoniae & $19(8.2)$ & $1(0.4)$ & 20.6 (2.7 to 155.3$)$ & $14.5(2.2$ to 94.8$)$ & $7.2(3.5$ to 10.8$)$ \\
\hline Chlamydophila pneumoniae & 0 & $1(0.4)$ & - & & \\
\hline At least one species of bacteria identified & $165(71.7)$ & $183(79.5)$ & $0.6(0.4$ to 1.0$)$ & $0.7(0.4$ to 1.2$)$ & \\
\hline One bacteria & $69(30.0)$ & $88(38.2)$ & & & \\
\hline Two or more bacterial species & $96(41.7)$ & $95(41.3)$ & & & \\
\hline
\end{tabular}

${ }^{*}$ Adjusted for age group, gender, prematurity, aboriginal status, comorbidity, childcare attendance, smoker at household, antibiotic exposure in last 7 days of enrolment and presence of other pathogens.

CAP, community-acquired pneumonia; HMPV, human metapneumovirus; HPIV, human parainfluenza virus; RSV, respiratory syncytial virus.

RSV was the most frequently detected virus in children with pneumonia and was most common in children $<2$ years. The proportion of children with pneumonia in whom RSV was detected was consistent with that reported in previous studies. ${ }^{8-10} 1928$ In contrast, few control children in our study were RSV positive (3\%), also consistent with the published literature. ${ }^{11} 142030$ Another member from the paramyxoviridae family, HMPV, was detected in $10 \%$ of cases but infrequently detected in healthy controls. The high adjusted OR for the detection of RSV and HMPV in children with pneumonia strongly implicates these viruses as probable pneumonia-causing pathogens. RSV is well established as a major cause of lower respiratory tract infection. ${ }^{31}$ This study adds to the growing evidence that HMPV is also an important respiratory pathogen in young children. ${ }^{911} 203233$
Children with pneumonia were also more likely to have influenza detected. Influenza is well documented to cause severe and occasionally fatal respiratory infection. ${ }^{34}$ All influenza-positive cases and controls were unvaccinated. Low influenza vaccination has been reported among children with influenza-confirmed pneumonia previously. ${ }^{35}$ Consistent with a protective effect, improved uptake of seasonal influenza vaccine in children is likely to reduce hospitalisations for pneumonia. RV was detected in similar proportions of children with (15\%) and without $(21 \%)$ pneumonia. Previous studies also detected RV in both children with pneumonia ${ }^{9112728}$ as well as in apparently asymptomatic children. ${ }^{1436}$ The latter raises doubt about the contribution of $\mathrm{RV}$ in pneumonia. ${ }^{37}$ Our finding is in line with other case-control studies that also failed to show the significant contribution of $\mathrm{RV}$ in acute respiratory infections in children. ${ }^{911}{ }^{38}$ While RV, 

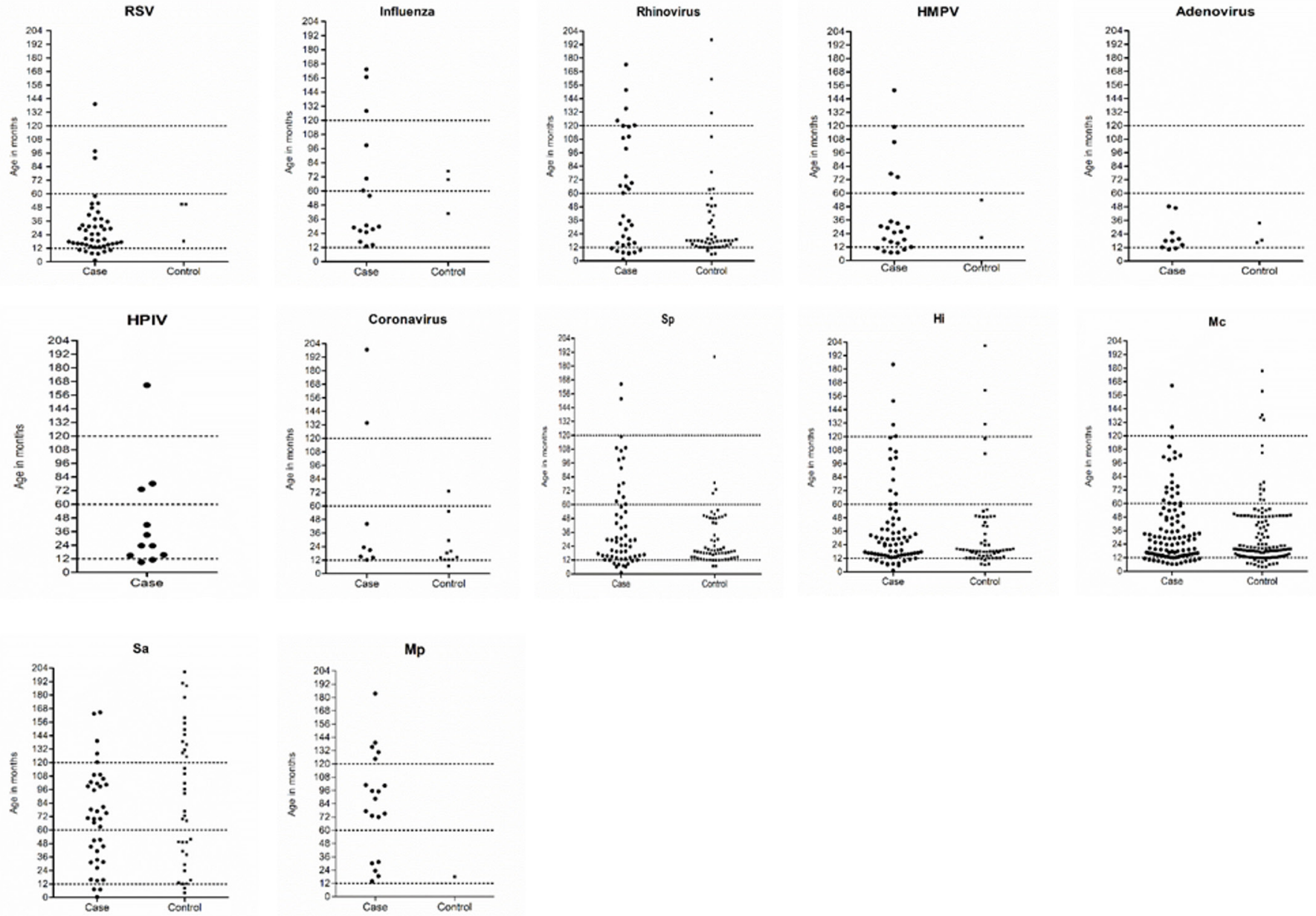

Figure 1 Distribution of respiratory pathogens by participant's age (in months); dot lines refers to age-group breakdown among study participants; for bacteria, such as Haemophilus influenzae (Hi), Streptococcus pneumoniae (Sp), Moraxella catarrhalis (Mc) and Staphylococcus aureus (Sau), only children without prior antibiotic exposure were included and Mycoplasma pneumoniae (Mp) included all children.

particularly RV-C, has been associated with childhood wheezing and asthma, ${ }^{39}$ its role in childhood pneumonia is not established. We had insufficient numbers to determine any specific RV types to be more prevalent among cases than controls; a study among Burundian children with lower respiratory tract symptoms associated RV-A with pneumonia and RV-C with wheezing. ${ }^{40}$ However, recently, using advanced molecular techniques such as transcriptomic analysis, a robust immune response had been measured in symptomatic children with detectable RV, substantially higher than in asymptomatic RV-positive children, ${ }^{41}$ suggesting the role of RV in acute respiratory infection needs more exploration.

M. pneumoniae was detected with similar frequency among cases to that reported in previous pneumonia studies and particularly prevalent among school-aged children and adolescents. ${ }^{81011}$ Unlike other studies, ${ }^{42} \mathrm{M}$. pneumoniae was identified infrequently in the nasopharynx of our control subjects. ${ }^{11} M$. pneumoniae most frequently causes non-severe atypical pneumonia. Detection of M. pneumoniae in the nasopharynx is reported in asymptomatic children, making it further difficult to interpret their clinical role in pneumonia. Moreover, there are conflicting and inconclusive reports on appropriate treatment for M. pneumoniae. Recent reviews and meta-analysis concluded that there is insufficient evidence on the efficacy of single or combination of multiple antibiotic for lower respiratory tract infections in children associated with M. pneumoniae. ${ }^{43-45}$
S. pneumoniae, H. influenzae and M. catarrhalis were detected with similar frequency in children with and without pneumonia. However, high vaccine coverage has almost eliminated pathogenic vaccine-type strains of $S$. pneumoniae and $H$. influenzae in this population. ${ }^{623}$ While there were no differences overall, S. pneumoniae was detected more frequently among infants with pneumonia than in control infants, suggesting S. pneumoniae may remain as an important cause of pneumonia in early life. While our results suggest a lesser contribution of bacteria to childhood pneumonia than viruses, we caution that we have not disproved an important role for bacteria. It is plausible, for example, that secondary bacterial infection plays an important role in many cases of viral pneumonia. ${ }^{34}$

A major strength of our study was the contemporaneous enrolment of children without pneumonia who were frequency-matched by age to pneumonia cases throughout the study period, which addressed methodological limitations in previous case-control studies in developed settings during post pneumococcal vaccination era. ${ }^{9}{ }^{11}$ However, our study has some limitations. To detect respiratory viruses and bacteria, we collected biological specimens from the upper respiratory tract (nasopharynx). The diagnosis of probable infectious agents associated with the lower respiratory tract illness such as pneumonia in children is often based on minimally invasive nasopharyngeal aspirates or nose/throat swabs. ${ }^{9} 46$ Testing of specimens from usually sterile sites (including blood and pleural fluid for culture 


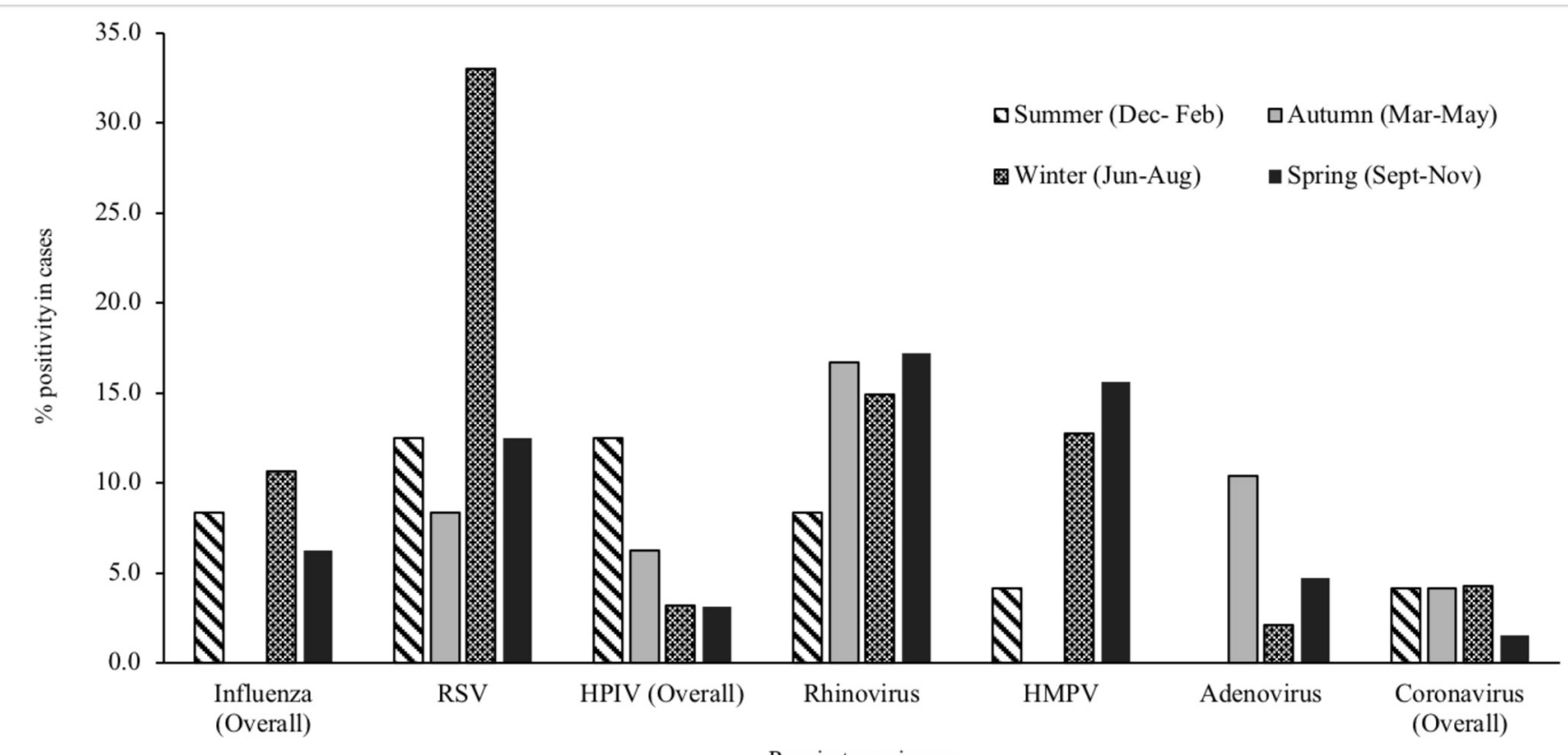

Figure 2 Distribution of respiratory viruses in the nasopharynx of children with community-acquired pneumonia (case group), by seasons, Perth, Western Australia, May 2015-October 2017. HMPV, human metapneumovirus; HPIV, human parainfluenza virus; RSV, respiratory syncytial virus.

or PCR) is available in our setting but was not mandated by our protocol. It is to be noted that while detecting a pathogen in the upper respiratory tract is not conclusive of its causal association with disease; however, testing control children without pneumonia enabled us to have a better estimation on contribution to pneumonia at the population level. Our ability to identify respiratory pathogens associated with pneumonia was limited by the sensitivity of testing the specimens from nasopharynx for infection in the lower airways. Differences between pathogen detection in the upper and lower tract have been shown previously ${ }^{47}$; it is plausible that in some cases with detectable virus, shedding of virus from the nasopharynx might be insignificant, or that shedding might be waning by the time children are hospitalised with pneumonia. Because of the sensitivity of our testing methods is likely incomplete, we expect that these estimates of the contribution of viruses to pneumonia represent minimum estimates. We used qualitative PCR assays in this study; determination of pathogen density through quantitative assays may

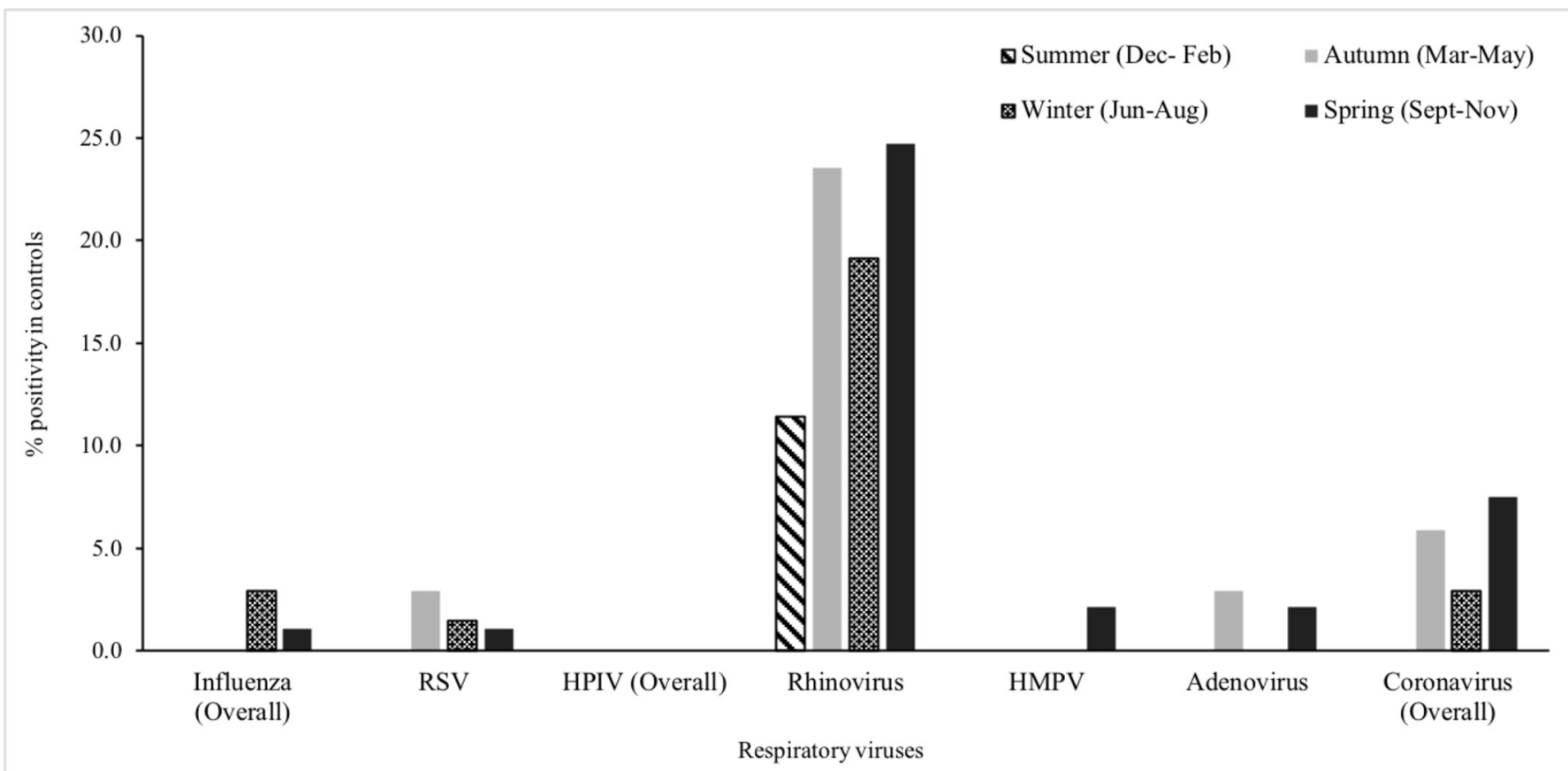

Figure 3 Distribution of respiratory viruses in the nasopharynx of healthy children (control group), by seasons, Perth, Western Australia, May 2015October 2017. HMPV, human metapneumovirus; HPIV, human parainfluenza virus; RSV, respiratory syncytial virus. 


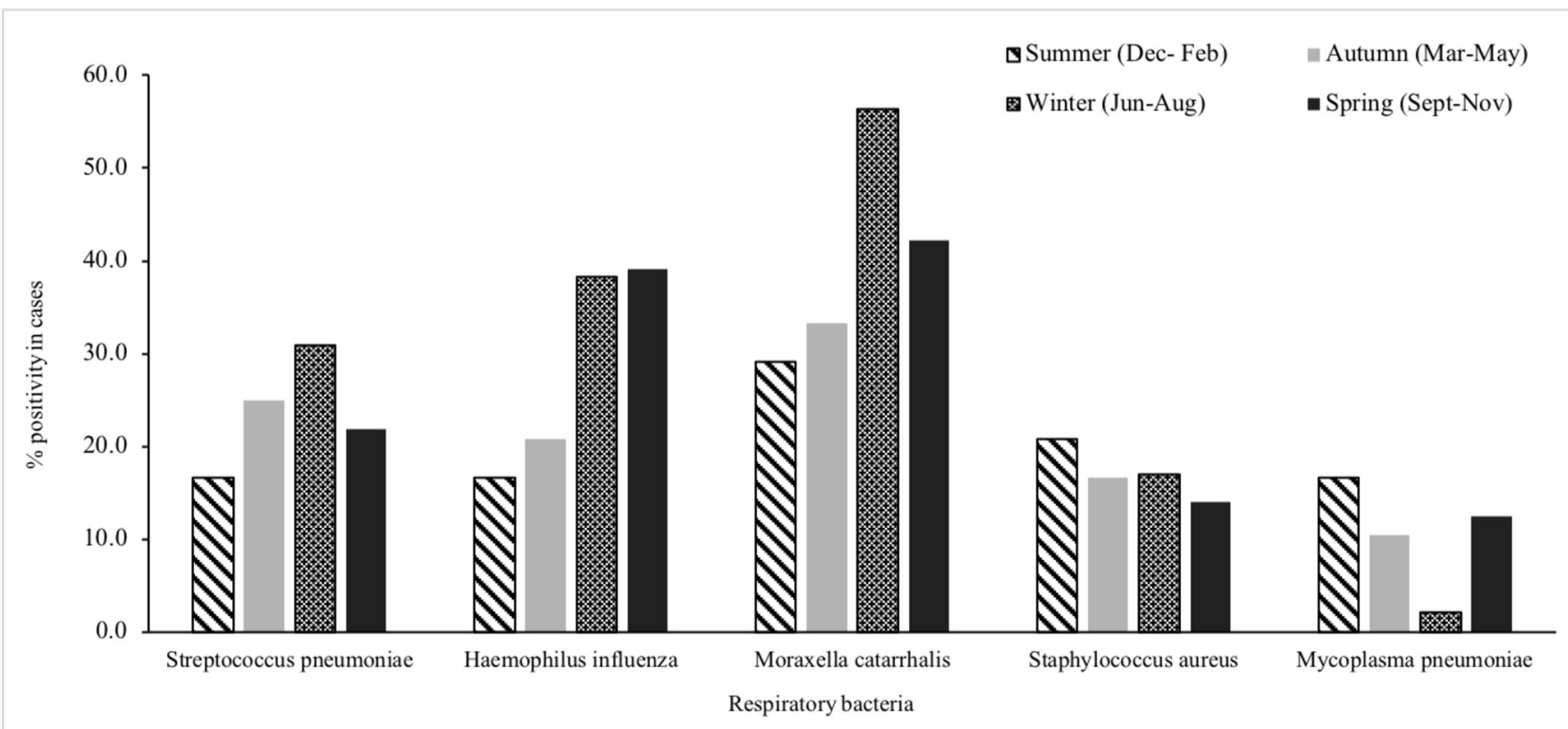

Figure 4 Distribution of bacteria in the nasopharynx of children with community-acquired pneumonia (case group), by seasons, Perth, Western Australia, May 2015-October 2017.

permit distinction of causative from coincidental infection in particular cases of pneumonia. ${ }^{48}$ We did not collect but data on radiographic details, for example, lobar consolidation or intestinal infiltrate, of enrolled cases would be useful to assess if radiographic pattern is associated with pathogen (bacterial or viral or both) detected in nasopharynx.

The burden of childhood pneumonia could be reduced if effective vaccines and treatments targeting viruses were readily available. ${ }^{49}{ }^{50}$ Understanding the contribution of each virus to pneumonia is important to informing and prioritising vaccine development. Australia has well-established health infrastructure with high rates of vaccine coverage. The findings of our study are expected to be generalisable to similar developed settings but not necessarily to resource-poor settings countries with high crowding, different social mixing patterns and low vaccine coverage. Nonetheless, we note that our data are comparable to data from case-control studies from both high-income and middle- and low-income countries. ${ }^{15} 2030$

This is the most comprehensive study that described contribution of respiratory pathogens to childhood pneumonia in Australia to date and one of few case-control studies on childhood pneumonia from high-income settings in the PCV era. We

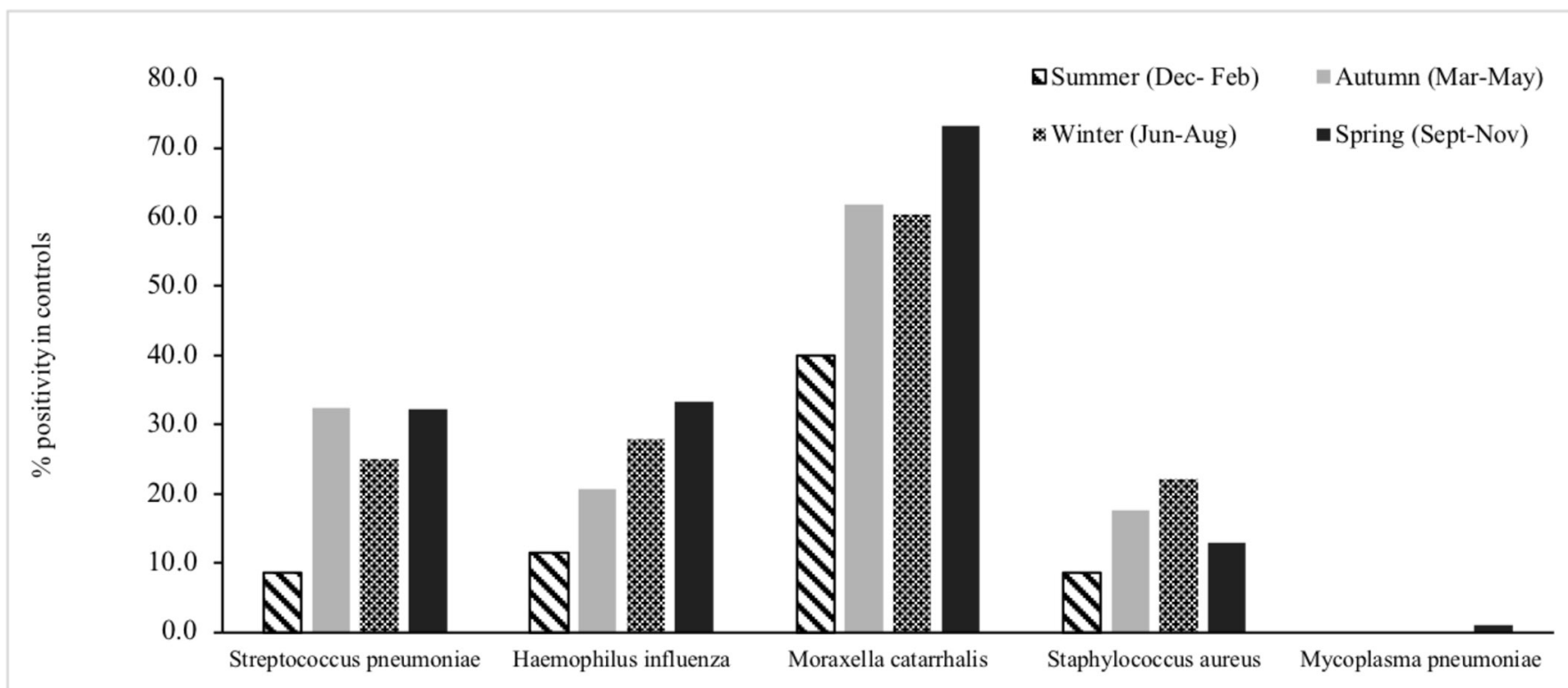

Respiraotry bacteria

Figure 5 Distribution of bacteria in the nasopharynx of healthy children (control group), by seasons, Perth, Western Australia, May 2015-0ctober 2017. 
found that respiratory viruses are a major contributor to childhood pneumonia. RSV appeared to account for a substantial proportion of viral pneumonia and should continue to be a focus for the development of preventative and treatment strategies. The burden of HMPV pneumonia may have been underappreciated before now. Data from this study could serve as baseline characteristics to assess the effectiveness of viral vaccines after their availability. Future studies should aim to develop a precise diagnostic tool that reliably distinguish viral from bacterial pneumonia to allow for targeted antiviral therapies and the more judicious use of antibiotics in treating childhood pneumonia.

\section{Author affiliations}

${ }^{1}$ Division of Paediatrics, School of Medicine, Faculty of Health and Medical Sciences, The University of Western Australia, Perth, Western Australia, Australia

${ }^{2}$ Wesfarmers Centre of Vaccines and Infectious Diseases, Telethon Kids Institute, Perth, Western Australia, Australia

${ }^{3}$ Department of Infectious Diseases, Perth Children's Hospital, Perth, Western Australia, Australia

${ }^{4}$ Department of Microbiology, PathWest Laboratory Medicine WA, Perth, Western Australia, Australia

${ }^{5}$ School of Biomedical Sciences, Faculty of Health and Medical Sciences, The University of Western Australia, Perth, Western Australia, Australia

${ }^{6}$ Emergency Department, Perth Children's Hospital, Perth, Western Australia, Australia ${ }^{7}$ Division of Emergency Medicine, School of Medicine, Faculty of Health and Medical Sciences, The University of Western Australia, Perth, Western Australia, Australia ${ }^{8}$ Department of General Paediatrics, Perth Children's Hospital, Perth, Western Australia, Australia

${ }^{9}$ School of Women's and Children's Health, Faculty of Medicine, University of New South Wales, Sydney, New South Wales, Australia

Acknowledgements The authors thank all the study participants and their parents for their participation in this study. The authors also thank nurses and physicians of the Emergency and General Paediatrics Departments and outpatient clinics of Princess Margaret Hospital for Children, staff of the local immunisation clinic, Perth, Western Australia, for helping in study participant recruitment. The authors also thank all nurses and research assistants of Vaccine Trial Group, Telethon Kids Institute who recruited study participants, and collected and processed study samples. Telethon Kids Institute acknowledges Aboriginal and Torres Strait Islander people as the Traditional Custodians of the land and waters of Australia. We also acknowledge the Nyoongar Wadjuk, Yawuru, Kariyarra and Kaurna Elders, their people and their land on which the Institute is located and seek their wisdom in our work to improve the health and development of all children.

Contributors MUB: Participant enrolment, data collection, specimen collection, data analysis, first draft manuscript and subsequent versions. TLS: Conceive the study, supervise data collection, supervise data analysis, critical review of manuscript. RW: Participant enrolment, data collection, specimen collection, critical review of manuscript. JL: Laboratory testing, critical review of manuscript. TR: Laboratory testing, critical review of manuscript. CG: Laboratory testing, critical review of manuscript. CdG: Laboratory testing, critical review of manuscript. MLB: Conceive the study, support data collection, critical review of manuscript. RBT: Supervise laboratory testing, interpret laboratory finding, critical review of manuscript. L-ASK: Supervise laboratory testing, interpret laboratory finding, critical review of manuscript. CS: Laboratory testing, critical review of manuscript. ACM: Conceive the study, support data collection, critical review of manuscript. PCR: Conceive the study, critical review of manuscript. DWS: Conceive the study, supervise laboratory testing, interpret laboratory finding, critical review of manuscript. AJ: Conceive the study, critical review of manuscript. CCB: Conceive the study, supervise data collection, interpret laboratory analysis, supervise data analysis, critical review of manuscript.

Funding Funding for the study has been provided by the Telethon-Perth Children's Hospital Research Fund, Perth Children's Hospital Foundation and Telethon Kids Institute. TLS and CCB are supported by NHMRC Career Development Fellowships.

Competing interests $P C R$ receives grants from GlaxoSmithKline, Novavax, Medimmune and Janssen outside the submitted work; L-ASK has a patent W02005108580A1 licensed to Pfizer.

Patient consent Written informed consent was obtained from parents/legal guardians of all study participants.

Ethics approval The protocol was approved by Human Research Ethics Committee of the Princess Margaret Hospital (PMH HREC REF 2014117EP).

Provenance and peer review Not commissioned; externally peer reviewed.

Open access This is an open access article distributed in accordance with the Creative Commons Attribution Non Commercial (CC BY-NC 4.0) license, which permits others to distribute, remix, adapt, build upon this work non-commercially, and license their derivative works on different terms, provided the original work is properly cited, appropriate credit is given, any changes made indicated, and the use is non-commercial. See: http://creativecommons.org/licenses/by-nc/4.0/.

\section{REFERENCES}

1 Walker CLF, Rudan I, Liu L, et al. Global burden of childhood pneumonia and diarrhoea. Lancet 2013:381:1405-16.

2 Prayle A, Atkinson M, Smyth A. Pneumonia in the developed world. Paediatr Respir Rev 2011:12:60-9.

3 Rudan I, Boschi-Pinto C, Biloglav Z, et al. Epidemiology and etiology of childhood pneumonia. Bull World Health Organ 2008;86:408-16.

4 Burgner D, Richmond P. The burden of pneumonia in children: an Australian perspective. Paediatr Respir Rev 2005;6:94-100.

5 Moore $H$, Burgner D, Carville K, et al. Diverging trends for lower respiratory infections in non-Aboriginal and Aboriginal children. J Paediatr Child Health 2007:43:451-7.

6 Fathima P, Blyth CC, Lehmann D, et al. The impact of pneumococcal vaccination on bacterial and viral pneumonia in western australian children: record linkage cohort study of 469589 births, 1996-2012. Clin Infect Dis 2018;66:1075-85.

7 Griffin MR, Mitchel E, Moore MR, et al. Declines in pneumonia hospitalizations of children aged $<2$ years associated with the use of pneumococcal conjugate vaccines Tennessee, 1998-2012. MMWR Morb Mortal Wkly Rep 2014;63:995-8.

8 Juvén T, Mertsola J, Waris M, et al. Etiology of community-acquired pneumonia in 254 hospitalized children. Pediatr Infect Dis J 2000;19:293-8.

9 Rhedin S, Lindstrand A, Hjelmgren A, et al. Respiratory viruses associated with community-acquired pneumonia in children: matched case-control study. Thorax 2015;70:847-53.

10 Cevey-Macherel M, Galetto-Lacour A, Gervaix A, et al. Etiology of community-acquired pneumonia in hospitalized children based on WHO clinical guidelines. Eur J Pediatr 2009;168:1429-36.

11 Jain S, Williams DJ, Arnold SR, et al. Community-acquired pneumonia requiring hospitalization among U.S. children. N Eng/ J Med 2015;372:835-45.

12 Nascimento-Carvalho AC, Vilas-Boas AL, Fontoura MH, et al. Respiratory viruses among children with non-severe community-acquired pneumonia: A prospective cohort study. J Clin Virol 2018;105:77-83

13 Jansen RR, Wieringa J, Koekkoek SM, et al. Frequent detection of respiratory viruses without symptoms: toward defining clinically relevant cutoff values. J Clin Microbiol 2011:49:2631-6

14 Moore HC, Jacoby P, Taylor A, et al. The interaction between respiratory viruses and pathogenic bacteria in the upper respiratory tract of asymptomatic Aboriginal and non-Aboriginal children. Pediatr Infect Dis J 2010;29:540-5.

15 Bénet T, Sánchez Picot V, Messaoudi M, et al. Microorganisms associated with pneumonia in children $<5$ years of age in developing and emerging countries: the gabriel pneumonia multicenter, prospective, case-control study. Clin Infect Dis 2017:65:604-12.

16 Neuzil KM. Progress toward a respiratory syncytial virus vaccine. Clin Vaccine Immunol 2016:23:186-8.

17 Karron RA, San Mateo J, Thumar B, et al. Evaluation of a live-attenuated human parainfluenza type 1 vaccine in adults and children. J Pediatric Infect Dis Soc 2015;4:e143-e146

18 Márquez-Escobar VA. Current developments and prospects on human metapneumovirus vaccines. Expert Rev Vaccines 2017;16:419-31.

19 Berkley JA, Munywoki P, Ngama M, et al. Viral etiology of severe pneumonia among Kenyan infants and children. JAMA 2010;303:2051-7.

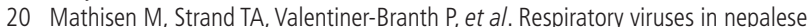
children with and without pneumonia: a case-control study. Pediatr Infect Dis J 2010;29:731-5

21 Bhuiyan MU, Snelling TL, West R, et al. Role of viral and bacterial pathogens in causing pneumonia among Western Australian children: a case-control study protocol. BMJ Open 2018:8:e020646.

22 Australian Bureau of Statistics, 2018. Australian demographic statistics, September 2017 http://www.abs.gov.au/Population (cited 5 Apr 2018).

23 National Centre For Immunisation Research \& Surveillance, 2016. Annual immunisation coverage report 2016 http://www.ncirs.edu.au/assets/surveillance/ coverage/NCIRS-Annual-Immunisation-Coverage-Report-2016.pdf (cited 2 Ap 2018).

24 Cherian T, Mulholland EK, Carlin JB, et al. Standardized interpretation of paediatric chest radiographs for the diagnosis of pneumonia in epidemiological studies. Bull World Health Organ 2005;83:353-9.

25 Lee WM, Lemanske RF, Evans MD, et al. Human rhinovirus species and season of infection determine illness severity. Am J Respir Crit Care Med 2012;186:886-91.

26 Greenland S, Drescher K. Maximum likelihood estimation of the attributable fraction from logistic models. Biometrics 1993;49:865-72.

27 Berg AS, Inchley CS, Aase A, et al. Etiology of pneumonia in a pediatric population with high pneumococcal vaccine coverage: a prospective study. Pediatr Infect Dis J 2016;35:e69-e75. 
28 Cilla G, Oñate E, Perez-Yarza EG, et al. Viruses in community-acquired pneumonia in children aged less than 3 years old: high rate of viral coinfection. J Med Virol 2008;80:1843-9.

29 Nascimento-Carvalho CM, Ribeiro CT, Cardoso MR, et al. The role of respiratory viral infections among children hospitalized for community-acquired pneumonia in a developing country. Pediatr Infect Dis J 2008;27:939-41.

30 Kelly MS, Smieja M, Luinstra K, et al. Association of respiratory viruses with outcomes of severe childhood pneumonia in Botswana. PLoS One 2015;10:e0126593.

31 Shi T, McAllister DA, O'Brien KL, et al. Global, regional, and national disease burden estimates of acute lower respiratory infections due to respiratory syncytial virus in young children in 2015: a systematic review and modelling study. Lancet 2017;390:946-58.

32 Ali A, Khowaja AR, Bashir MZ, et al. Role of human metapneumovirus, influenza A virus and respiratory syncytial virus in causing WHO-defined severe pneumonia in children in a developing country. PLoS One 2013;8:e74756.

33 Nascimento-Carvalho CM, Cardoso MR, Ruuskanen O, et al. Sole infection by human metapneumovirus among children with radiographically diagnosed community-acquired pneumonia in a tropical region. Influenza Other Respir Viruses 2011:5:285-7.

34 Blyth CC, Webb SA, Kok J, et al. The impact of bacterial and viral co-infection in severe influenza. Influenza Other Respir Viruses 2013;7:168-76.

35 Grijalva CG, Zhu Y, Williams DJ, et al. Association between hospitalization with community-acquired laboratory-confirmed influenza pneumonia and prior receipt of influenza vaccination. JAMA 2015;314:1488-97.

36 Jartti T, Jartti L, Peltola V, et al. Identification of respiratory viruses in asymptomatic subjects: asymptomatic respiratory viral infections. Pediatr Infect Dis J 2008;27:1103-7.

37 Lu QB, Wo Y, Wang LY, et al. Molecular epidemiology of human rhinovirus in children with acute respiratory diseases in Chongqing, China. Sci Rep 2014;4:6686.

38 Singleton RJ, Bulkow LR, Miernyk K, et al. Viral respiratory infections in hospitalized and community control children in Alaska. J Med Virol 2010;82:1282-90.
39 Bizzintino J, Lee WM, Laing IA, et al. Association between human rhinovirus $C$ and severity of acute asthma in children. Eur Respir J 2011;37:1037-42.

40 Esposito S, Daleno C, Baggi E, et al. Circulation of different rhinovirus groups among children with lower respiratory tract infection in Kiremba, Burundi. Eur J Clin Microbiol Infect Dis 2012;31:3251-6.

41 Heinonen S, Jartti T, Garcia C, et al. Rhinovirus detection in symptomatic and asymptomatic children: value of host transcriptome analysis. Am J Respir Crit Care Med 2016;193:772-82

42 Spuesens EB, Fraaij PL, Visser EG, et al. Carriage of Mycoplasma pneumoniae in the upper respiratory tract of symptomatic and asymptomatic children: an observational study. PLoS Med 2013;10:e1001444

43 Biondi E, McCulloh R, Alverson B, et al. Treatment of mycoplasma pneumonia: a systematic review. Pediatrics 2014;133:1081-90.

44 Blyth CC, Gerber JS. Macrolides in children with community-acquired pneumonia: panacea or placebo? J Pediatric Infect Dis Soc 2018:7:71-7.

45 Gardiner SJ, Gavranich JB, Chang AB. Antibiotics for community-acquired lower respiratory tract infections secondary to Mycoplasma pneumoniae in children. Cochrane Database Syst Rev 2015;1:CD004875.

46 Hammitt LL, Murdoch DR, Scott JA, et al. Specimen collection for the diagnosis of pediatric pneumonia. Clin Infect Dis 2012;54(Suppl 2):S132-9.

47 Blyth CC, Iredell JR, Dwyer DE. Rapid-test sensitivity for novel swine-origin influenza A (H1N1) virus in humans. N Engl J Med 2009;361:2493.

48 Feikin DR, Fu W, Park DE, et al. Is Higher viral load in the upper respiratory tract associated with severe pneumonia? Findings from the PERCH Study. Clin Infect Dis 2017;64(suppl 3):S337-\$346.

49 The IMpact-RSV Study Group,. Palivizumab, a humanized respiratory syncytial virus monoclonal antibody, reduces hospitalization from respiratory syncytial virus infection in high-risk infants. The IMpact-RSV Study Group. Pediatrics 1998;102:531-7.

50 Heinonen $\mathrm{S}$, Silvennoinen $\mathrm{H}$, Lehtinen $\mathrm{P}$, et al. Effectiveness of inactivated influenza vaccine in children aged 9 months to 3 years: an observational cohort study. Lancet Infect Dis 2011;11:23-9. 